\title{
Validation of the Polish version of the Unified Dyskinesia Rating Scale (UDysRS)
}

\author{
Joanna Siuda $^{1 \star}$, Magdalena Boczarska-Jedynak ${ }^{2 \star}$, Sławomir Budrewicz ${ }^{3}$, Jarosław Dulski ${ }^{4,5}$, \\ Monika Figura $^{6}$, Urszula Fiszer ${ }^{7}$, Agata Gajos ${ }^{8}$, Agnieszka Gorzkowska ${ }^{9 \star}$, Ewa Koziorowska-Gawron ${ }^{3}$, \\ Dariusz Koziorowski ${ }^{6}$, Anna Krygowska-Wajs ${ }^{10}$, Monika Rudzińska-Bar ${ }^{11}$, Jarosław Sławek ${ }^{4,5}$, \\ Mateusz Toś, Magdalena Wójcik-Pędziwiatr ${ }^{11}$, Jeffery Lin $^{12}$, Sheng Luo ${ }^{13}$, \\ Pablo Martinez-Martin ${ }^{14}$, Glenn T. Stebbins ${ }^{15}$, Christopher G. Goetz ${ }^{15}$, Grzegorz Opala ${ }^{1}$, \\ and the Polish UDysRS Validation Task Force ${ }^{* *}$ \\ ${ }^{1}$ Department of Neurology, Faculty of Medical Sciences in Katowice, Medical University of Silesia, Katowice, Poland \\ ${ }^{2}$ Department of Neurology and Regenerative Medicine, Health Institute dr Boczarska-Jedynak, Oświęcim, Poland \\ ${ }^{3}$ Department of Neurology, Wroclaw Medical University, Wroclaw, Poland \\ ${ }^{4}$ Department of Neurological and Psychiatric Nursing, Faculty of Health Sciences, Medical University of Gdansk, Gdansk, Poland \\ ${ }^{5}$ Department of Neurology and Stroke, St. Adalbert Hospital, Gdansk, Poland \\ ${ }^{6}$ Department of Neurology, Faculty of Health Sciences, Medical University of Warsaw, Warsaw, Poland \\ ${ }^{7}$ Department of Neurology and Epileptology, Centre of Postgraduate Medical Education, Orłowski Hospital, Warsaw, Poland \\ ${ }^{8}$ Department of Extrapyramidal Diseases, Medical University of Łódź, Łódź, Poland \\ ${ }^{9}$ Department of Neurorehabilitation, Faculty of Medical Sciences in Katowice, Medical University of Silesia, Katowice, Poland \\ ${ }^{10}$ Department of Neurology, Jagiellonian University, Collegium Medicum, Krakow, Poland \\ ${ }^{11}$ Department of Neurology, Faculty of Medicine and Health Sciences, Andrzej Frycz Modrzewski Krakow University, Krakow, Poland \\ ${ }^{12}$ Department of Biostatistics, Gilead Sciences, Inc., Foster City, California, United States \\ ${ }^{13}$ Department of Biostatistics and Bioinformatics, Duke University, Durham, North Carolina, United States \\ ${ }^{14}$ Centre for Networked Biomedical Research in Neurodegenerative Diseases (CIBERNED), Carlos III Institute of Health, Madrid, Spain \\ ${ }^{15}$ Department of Neurological Sciences, Rush University Medical Centre, Chicago, Illinois, United States \\ ${ }^{16}$ Department of Neurology, John Paul II Specialised Hospital, Krakow, Poland
}

\section{ABSTRACT}

Background. In 2008, the Movement Disorders Society published the Unified Dyskinesia Rating Scale (UDysRS). This has become the established tool for assessing the severity and disability associated with dyskinesia in patients with Parkinson's Disease (PD). We translated and validated the Polish version of the UDysRS, explored its dimensionality, and compared it to the Spanish version, which is the Reference Standard for UDysRS translations.

Materials and methods. The UDysRS was translated into Polish by a team led by JS and GO. The back-translation, completed by colleagues fluent in both Polish and English who were not involved in the original translation, was reviewed and approved by the Executive Committee of the MDS Rating Scales Programme. Then the translated version of the UDysRS underwent cognitive pretesting, and the translation was modified based on the results. The approved version was considered to be the Official Working Document of the Polish UDysRS and was tested on 250 Polish PD patients recruited at movement disorder centres. Data was compared to the Reference Standard used for validating UDysRS translations.

\footnotetext{
* translation/back-translation team members

** Task Force members in alphabetical order: Andrzej Bogucki ${ }^{8}$, Piotr Janik ${ }^{6}$, Magdalena Koszewicz ${ }^{3}$, Marta Leńska-Mieciek ${ }^{7}$, Małgorzata Michałowska $^{7}$, Marta Piaścik-Gromada ${ }^{7}$, Katarzyna Potasz-Kulikowska ${ }^{10}$, Marek Śmiłowski ${ }^{*}$, Anna Wasilewska ${ }^{16}$
}

Address for correspondence: Joanna Siuda, Department of Neurology, Faculty of Medical Sciences in Katowice, Medical University of Silesia, Katowice, Poland, e-mail: jsiuda@sum.edu.pl 
Results. The overall factor structure of the Polish version was consistent with that of the Reference Standard version, as evidenced by the high Confirmatory Fit Index score $(\mathrm{CFI}=0.98)$. The Polish UDysRS was thus confirmed to share a common factor structure with the Reference Standard.

Conclusions. The Official Polish UDysRS translation is recommended for use in clinical and research settings. Worldwide use of uniform rating measures offers a common ground to study similarities and differences in disease manifestations and progression across cultures.

Key words: Parkinson's Disease, dyskinesia, validation, translation, rating scales, UDysRS

(Neurol Neurochir Pol 2021; 55 (2): 186-194)

\section{Introduction}

In advanced Parkinson's Disease (PD), among many disabling symptoms (motor fluctuations, autonomic dysfunction, cognitive and psychiatric disorders), drug-induced dyskinesia (DID) is one of the most difficult to manage [1-4]. It is important to recognise and objectively assess the severity of DID in PD [5].

In this regard, the International Parkinson and Movement Disorder Society (MDS) in 2008 published a comprehensive rating tool of dyskinesia in PD: the Unified Dyskinesia Rating Scale (UDysRS). Today, the UDysRS is commonly used to assess dyskinesia severity and associated disability. UDysRS combines patient-based assessments of dyskinesia with objective evaluations of disability and impairment from dyskinesias, and it has been clinimetrically validated [6].

The UDysRS is composed of four parts:

- Part 1: ON Dyskinesia - measures the subjective impact of ON dyskinesia on everyday activities. The first item, on time spent with ON dyskinesia, is assessed by a trained rater (Part $1 \mathrm{~A})$, whereas the remaining 10 items (Part $1 \mathrm{~B}$ ) are self-rated by the patient and/or given from the caregiver's perspective (11 items, giving a maximum of 44 points);

- Part 2: OFF Dystonia - evaluates the burden caused by OFF dystonia. The first item, on time spent with OFF dystonia, is evaluated by a trained rater (Part $2 \mathrm{~A}$ ), and the remaining three items (Part $2 \mathrm{~B}$ ) are information from the patient and/or the caregiver's perspective (four items, giving a maximum of 16 points);

- Part 3: Impairment - objectively assesses dyskinesia severity, anatomical distribution over seven body regions, and type (i.e. choreic and/or dystonic) based on four activities observed during a clinical life examination or video recorded (seven items, giving a maximum of 28 points);

- Part 4: Disability - examines the disability associated with dyskinesia on four representative tasks, including communication, drinking, dressing, and ambulation (four items, giving a maximum of 16 points).

To enhance the uniform administration of the UDysRS, the MDS Rating Scales Programme sets a specific protocol to designate successful translation of non-English versions [7].
We aimed to translate and validate a Polish version of the UDysRS scale, and to compare it to the Spanish language version, which is the established Reference Standard for UDysRS translations [8].

Below, we present a scale translation and clinimetric testing results of the Polish version of the UDysRS.

\section{Materials and methods}

The participants were $250 \mathrm{PD}$ patients recruited from the neurology departments in ten sites across Poland (two in Katowice, two in Krakow, one in Wroclaw, three in Warsaw, one in Gdansk, and one in Lodz). At each site, experienced Polish-speaking movement disorder specialists were recruited to examine native Polish-speaking PD patients with different distributions and severities of dyskinesia. All patients participated voluntarily and gave written informed consent prior to the study. Anonymised data, without patient names or medical record numbers, was transferred to the analytic team via a secure website. The Reference Standard for UDysRS translations is the Spanish language version of the scale, validated previously on 253 native Spanish-speaking PD patients [8]. This data available from the MDS Translation Committee was used for comparative analysis of the UDysRS according to the MDS-established Protocol for official non-English language translations.

\section{Procedure}

The MDS Rating Scales Programme has prepared a well-defined protocol, with objective criteria for translation and validation of non-English versions of the MDS-UPDRS and UDysRS in order to have an 'official' MDS translation in a foreign language [7].

There is a four-step process involved in developing an officially approved translation of these scales: (1) translation and independent back-translation; (2) cognitive pretesting to establish that the translation is clear, comfortably administered by native-speaker raters, and understood by native-speaker patients; (3) field testing in the native language using a large sample of PD patients; and (4) statistical analyses including validity testing and factor analysis. This process was previously used by our team in a successful validation of thevPolish version of the MDS-UPDRS [9]. 


\section{Translation of UDysRS}

This Polish version validation was performed as follows: firstly, the UDysRS was translated into Polish by a team of Polish speakers who were not only fluent in English but were also physicians and specialists in movement disorders. This team was led by Dr. Joanna Siuda and Prof. Grzegorz Opala. This was then back-translated into English by colleagues fluent in both English and Polish who had not been involved in the original translation. Finally, it was reviewed by a team of American experts led by Profs. Christopher Goetz and Glenn Stebbins (of the Executive Translation Programme Committee, ETPC) who had been involved in the development of the original English language version [6].

\section{Cognitive pretesting}

Cognitive pretesting is a qualitative approach to assessing task difficulty for examiner and respondent, respondent interest, attention span, discomfort, and comprehension [10].

Where there were observed differences between the back-translated Polish version and the English version, items were selected for cognitive pretesting along with questions that were identified during cognitive pretesting of the English version.

The question topics included in cognitive pretesting were: Instructions to Raters and Instructions to Patients; Time Spent with Dyskinesia; Chewing and Swallowing; Exciting or Emotional Settings; Effects of Spasms or Cramps Separate from Pain on Activities; Objective Impairment Ratings; and Objective Disability Ratings.

Based on the results of the initial cognitive pretesting, other round(s) of translation and back translation and cognitive pretesting may be required. Once cognitive pretesting responses were taken into account, the version was modified into the final translation that was approved by the ETPC.

\section{Factor analysis}

To conduct the factor analysis of the UDysRS, we omitted Questions 1 (time of ON dyskinesia) and 12 (time of OFF dystonia) and considered these items as descriptive indices rather than measures of impairment or disability. To maximise the accuracy of these time indices, we added three clarifying statements to ensure harmonisation of the time-based questions with the patient/caregiver questionnaire and interview items: In the initial instructions to the full scale, we alert the rater to review the patient questionnaire after completion to ensure that, if item scores indicate the presence of dyskinesia or dystonia over the past week, the time-based items also reflect their occurrence (rating 1, 2, 3, or 4 but not 0 ).

At the end of each questionnaire section (ON dyskinesia and OFF dystonia), the same alert is inserted.

M-plus Version 7.4 was used to carry out the confirmatory and exploratory factor analyses as the variables are categorical [11]. We used the weighted least squares (WLSMV) approach to factor estimation that minimises the weighted sum of squared differences between observed and estimated correlation matrices. To assist in the interpretation of factors, we used an orthogonal VARIMAX rotation that constrains the factors to be uncorrelated.

The sample size for the translation study was based on the need for 7-10 subjects per item of the questionnaire in order to perform the tasks needed to validate the instrument [12].

Because there are 26 items on the UDysRS, a sample of at least 250 was required. The investigators obtained approval from the human subjects prior to data collection. Deidentified data (with no patient names or medical record numbers) was transferred to the analytic team via a secure website.

\section{Primary analysis}

As the primary analysis, we conducted a confirmatory factor analysis (CFA), comparing the Polish data to the Reference Standard data $[13,14]$. We determined whether the factor structure for the Spanish language UDysRS, which served as the Reference Standard, could be confirmed in the data collected using the Polish translation. This was the primary question of interest. We evaluated the CFA results based on the Comparative Fit Index (CFI). To confirm a good fit between the Polish and the Reference Standard UDysRS, we required that the CFI was 0.90 or greater. Mean and variance adjusted weighted least square (WLSMV) estimator was used to confirm model fit.

We also used the root mean square error of approximation (RMSEA) to check the goodness of fit. This is a populationbased index that relies on noncentral $\chi 2$ distribution, which is the distribution of the fitting function when the fit of the model is not perfect.

\section{Secondary analysis}

As a secondary analysis, we conducted an exploratory factor analysis (EFA) to explore the underlying factor structure for the Polish language translation, without constraint of a prespecified factor structure, using a weighted least squares (WLSMV) approach. We used a scree plot to choose the number of factors retained for UDysRS. The subjective scree test is a scatter plot of eigenvalues plotted against their ranks with respect to magnitude, to extract as many factors as there are eigenvalues that fall before the last large drop (i.e. an 'elbow' shape) in the plot [15]. Once the factors were chosen, an item was retained in a factor if the factor loading for the item was 0.40 or greater. To assist interpretation of the factors, an orthogonal CF-VARIMAX rotation was used which set the factors to be uncorrelated.

\section{Ethics}

All patients gave written consent to participate. The anonymised patient data was transferred to the US team for analysis via a secure website. The programme for validation of the UDysRS Polish version was approved for all sites by the Ethics Committee of the Medical University of Silesia in Katowice (KNW/0022/KB/121/14). 


\section{Results}

\section{Baseline characteristics}

The demographic characteristics of the Polish patients are set out in Table 1. The Polish dataset included 250 native Polish-speaking Parkinson's Disease patients with dyskinesia who were examined using the UDysRS. Table 2 sets out the distributions of answers to each question.

\section{Cronbach's alpha index and correlation analysis}

The overall raw and standardised Cronbach's coefficient alpha was 0.938 and 0.94 , respectively, indicating that the Polish UDysRS was reliable. Table 3 sets out the correlation between each question and the total score. Examination of the correlation of individual items to the total score revealed lower correlations for items 14 and 15.

\section{Cognitive pretesting}

Three examiners and 10 patients with Parkinson's Disease were interviewed using a structured cognitive pretesting manual. On the first round of cognitive pretesting, a few minor issues were identified for the instructions for the raters and for one definition provided to the patients. Slight modifications were made to the translation based on this feedback, and a second round of cognitive pretesting was requested with a new set of five patients. No problems were identified on the second round of testing by either patients or raters, so the translated scale was approved as the Official Working Document of the Polish UDysRS for testing in a larger group of patients with PD.
Primary analysis: Confirmatory Factor Analysis (CFA)

$\mathrm{M}$ plus performs listwise deletion of cases with any missing data. That is, any case with one or more missing data points is omitted entirely from analyses. Thus, the sample size in factor analysis is 250 . The Comparative Fit Index (CFI), compared to the Reference Standard factor structure, was 0.954, and root mean square error of approximation was 0.115 . Our pre-specified criterion was a CFI of 0.90 or greater.

Hence, we concluded that the pre-specified Reference Standard factor structure was confirmed in the Polish dataset.

Secondary analysis: Exploratory Factor Analysis (EFA)

Table 4 shows the results of Exploratory Factor Analysis for all patients of the Reference Standard and Polish UDysRS without the items for Time Spent with On Dyskinesia, and Time Spent with Off Dystonia. The scree plots are given in Figure 1. From these we extracted three factors. The factor structure of Polish UDysRS is quite consistent with that of Reference Standard UDysRS.

EFA analysis for the Polish UDysRS dataset differed from the EFA of the Reference Standard dataset in some areas. Two of the 24 items loaded on different factors in the two scales. In contrast to the Reference Standard UDysRS, Exciting situations loaded in the Polish version on factor 3, instead of factor 1 . Dystonia pain severity loaded on factor 2 in the Polish version, and on factor 3 in the Reference Standard. Ambulation did not load on any of the factors in the Polish version, but originally loaded on factor 2 in the Reference Standard. Communication

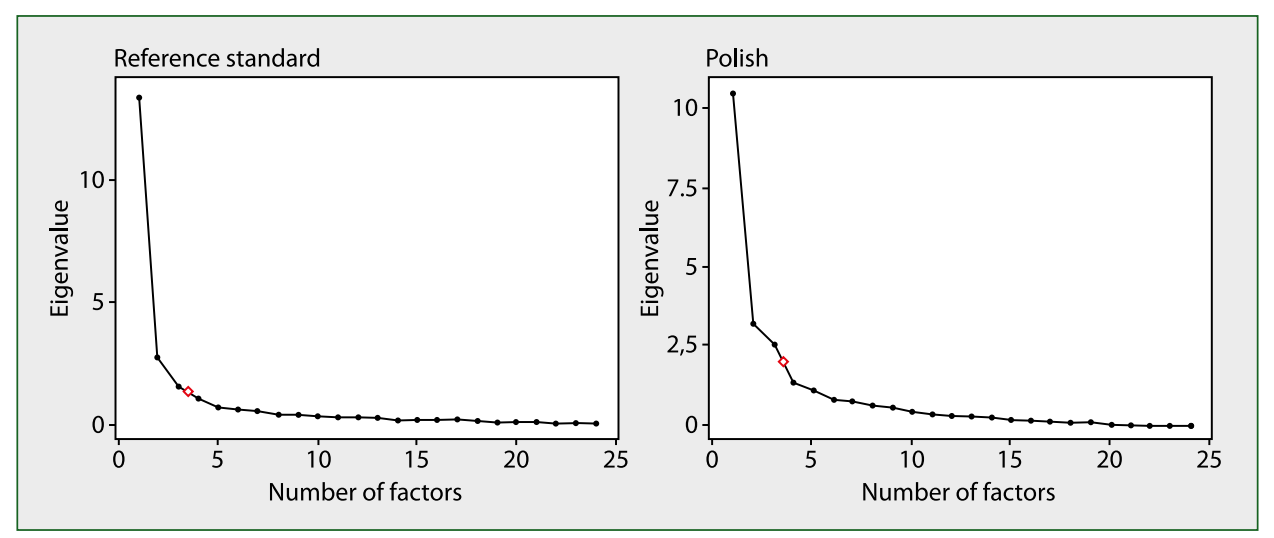

Figure 1.Scree plots for reference standard and Polish exploratory factor analyses

Table 1. Demographic characteristics of study population

\begin{tabular}{|c|c|c|c|c|c|c|c|c|c|}
\hline \multirow[t]{2}{*}{ Number of PD patients } & \multirow[b]{2}{*}{$\mathbf{N}$} & \multicolumn{2}{|c|}{ Male* } & \multicolumn{2}{|c|}{$\begin{array}{l}\text { Age** } \\
\text { (years) }\end{array}$} & \multicolumn{2}{|c|}{$\begin{array}{l}\text { PD duration*** } \\
\text { (years) }\end{array}$} & \multicolumn{2}{|c|}{$\begin{array}{c}\text { DID duration } * * * * \\
\text { (years) }\end{array}$} \\
\hline & & $\mathbf{N}$ & $\%$ & Mean & SD & Mean & SD & Mean & SD \\
\hline Reference standard & 253 & 122 & 48.2 & 69.2 & 10.5 & 12.5 & 6.8 & 4.9 & 4.6 \\
\hline Polish & 250 & 135 & 59 & 74.6 & 13.9 & 12.1 & 6 & 4.2 & 3 \\
\hline
\end{tabular}

*Data available for 229, **221, ***180, and ****171 subjects in Polish PD population; PD — Parkinson's Disease; DID — drug induced dyskinesia; SD — standard deviation 
Table 2. Distribution of UDysRS responses

\begin{tabular}{|c|c|c|c|c|c|c|c|c|c|}
\hline \multirow[t]{2}{*}{ Time dyskinesia } & \multicolumn{2}{|c|}{ Reference standard } & \multicolumn{2}{|c|}{ Polish } & \multirow[b]{2}{*}{ Speech } & \multicolumn{2}{|c|}{ Reference standard } & \multicolumn{2}{|c|}{ Polish } \\
\hline & Freq & Percent & Freq & Percent & & Freq & Percent & Freq & Percent \\
\hline 0 & 45 & 17.79 & 5 & 2 & 0 & 92 & 36.36 & 105 & 42 \\
\hline 1 & 56 & 22.13 & 101 & 40.4 & 1 & 99 & 39.13 & 81 & 32.4 \\
\hline 2 & 55 & 21.74 & 83 & 33.2 & 2 & 52 & 20.55 & 52 & 20.8 \\
\hline 3 & 37 & 14.62 & 43 & 17.2 & 3 & 8 & 3.16 & 9 & 3.6 \\
\hline 4 & 60 & 23.72 & 18 & 7.2 & 4 & 2 & 0.79 & 3 & 1.2 \\
\hline Total & 253 & 100 & 250 & 100 & Total & 253 & 100 & 250 & 100 \\
\hline $\begin{array}{l}\text { Chewing } \\
\text { /swallowing }\end{array}$ & Freq & Percent & Freq & Percent & Eating tasks & Freq & Percent & Freq & Percent \\
\hline 0 & 117 & 46.25 & 118 & 47.2 & 0 & 84 & 33.2 & 73 & 29.2 \\
\hline 1 & 84 & 33.2 & 88 & 35.2 & 1 & 83 & 32.81 & 81 & 32.4 \\
\hline 2 & 37 & 14.62 & 32 & 12.8 & 2 & 65 & 25.69 & 56 & 22.4 \\
\hline 3 & 14 & 5.53 & 10 & 4 & 3 & 18 & 7.11 & 26 & 10.4 \\
\hline 4 & 1 & 0.4 & 2 & 0.8 & 4 & 3 & 1.19 & 14 & 5.6 \\
\hline Total & 253 & 100 & 250 & 100 & Total & 253 & 100 & 250 & 100 \\
\hline Dressing & Freq & Percent & Freq & Percent & Hygiene & Freq & Percent & Freq & Percent \\
\hline 0 & 73 & 28.85 & 52 & 20.8 & 0 & 86 & 33.99 & 57 & 22.8 \\
\hline 1 & 71 & 28.06 & 88 & 35.2 & 1 & 81 & 32.02 & 88 & 35.2 \\
\hline 2 & 74 & 29.25 & 67 & 26.8 & 2 & 59 & 23.32 & 68 & 27.2 \\
\hline 3 & 25 & 9.88 & 32 & 12.8 & 3 & 20 & 7.91 & 24 & 9.6 \\
\hline 4 & 8 & 3.16 & 11 & 4.4 & 4 & 7 & 2.77 & 13 & 5.2 \\
\hline Total & 251 & 99.21 & 250 & 100 & Total & 253 & 100 & 250 & 100 \\
\hline Handwriting & Freq & Percent & Freq & Percent & $\begin{array}{l}\text { Doing hobbies/ } \\
\text { /activities }\end{array}$ & Freq & Percent & Freq & Percent \\
\hline 0 & 80 & 31.62 & 56 & 22.4 & 0 & 75 & 29.64 & 66 & 26.4 \\
\hline 1 & 65 & 25.69 & 74 & 29.6 & 1 & 80 & 31.62 & 74 & 29.6 \\
\hline 2 & 59 & 23.32 & 61 & 24.4 & 2 & 57 & 22.53 & 70 & 28 \\
\hline 3 & 38 & 15.02 & 42 & 16.8 & 3 & 29 & 11.46 & 28 & 11.2 \\
\hline 4 & 8 & 3.16 & 17 & 6.8 & 4 & 12 & 4.74 & 12 & 4.8 \\
\hline Total & 250 & 98.81 & 250 & 100 & Total & 253 & 100 & 250 & 100 \\
\hline Walking/balance & Freq & Percent & Freq & Percent & Public/social & Freq & Percent & Freq & Percent \\
\hline 0 & 69 & 27.27 & 46 & 18.4 & 0 & 67 & 26.48 & 21 & 8.4 \\
\hline 1 & 74 & 29.25 & 73 & 29.2 & 1 & 84 & 33.2 & 54 & 21.6 \\
\hline 2 & 68 & 26.88 & 80 & 32 & 2 & 62 & 24.51 & 88 & 35.2 \\
\hline 3 & 31 & 12.25 & 40 & 16 & 3 & 34 & 13.44 & 67 & 26.8 \\
\hline 4 & 11 & 4.35 & 11 & 4.4 & 4 & 6 & 2.37 & 20 & 8 \\
\hline Total & 253 & 100 & 250 & 100 & Total & 253 & 100 & 250 & 100 \\
\hline $\begin{array}{l}\text { Exciting } \\
\text { situations }\end{array}$ & Freq & Percent & Freq & Percent & Time off dystonia & Freq & Percent & Freq & Percent \\
\hline 0 & 72 & 28.46 & 22 & 8.8 & 0 & 125 & 49.41 & 78 & 31.2 \\
\hline 1 & 83 & 32.81 & 59 & 23.6 & 1 & 45 & 17.79 & 67 & 26.8 \\
\hline 2 & 59 & 23.32 & 82 & 32.8 & 2 & 27 & 10.67 & 55 & 22 \\
\hline 3 & 36 & 14.23 & 64 & 25.6 & 3 & 15 & 5.93 & 25 & 10 \\
\hline 4 & 2 & 0.79 & 23 & 9.2 & 4 & 40 & 15.81 & 25 & 10 \\
\hline Total & 252 & 99.6 & 250 & 100 & Total & 252 & 99.6 & 250 & 100 \\
\hline
\end{tabular}


Table 2 cont. Distribution of UDysRS responses

\begin{tabular}{|c|c|c|c|c|c|c|c|c|c|}
\hline \multirow{2}{*}{$\begin{array}{l}\text { Dystonia effects } \\
\text { on activities }\end{array}$} & \multicolumn{2}{|c|}{ Reference standard } & \multicolumn{2}{|c|}{ Polish } & \multirow[b]{2}{*}{$\begin{array}{l}\text { Effect of pain } \\
\text { from dystonia }\end{array}$} & \multicolumn{2}{|c|}{ Reference standard } & \multicolumn{2}{|c|}{ Polish } \\
\hline & Freq & Percent & Freq & Percent & & Freq & Percent & Freq & Percent \\
\hline 0 & 128 & 50.59 & 92 & 36.8 & 0 & 151 & 59.68 & 118 & 47.2 \\
\hline 1 & 45 & 17.79 & 60 & 24 & 1 & 34 & 13.44 & 51 & 20.4 \\
\hline 2 & 31 & 12.25 & 58 & 23.2 & 2 & 29 & 11.46 & 39 & 15.6 \\
\hline 3 & 29 & 11.46 & 26 & 10.4 & 3 & 29 & 11.46 & 32 & 12.8 \\
\hline 4 & 20 & 7.91 & 14 & 5.6 & 4 & 9 & 3.56 & 10 & 4 \\
\hline Total & 253 & 100 & 250 & 100 & Total & 252 & 99.6 & 250 & 100 \\
\hline $\begin{array}{l}\text { Dystonia pain } \\
\text { severity }\end{array}$ & Freq & Percent & Freq & Percent & Face & Freq & Percent & Freq & Percent \\
\hline 0 & 147 & 58.1 & 111 & 44.4 & 0 & 122 & 48.22 & 134 & 53.6 \\
\hline 1 & 21 & 8.3 & 52 & 20.8 & 1 & 73 & 28.85 & 51 & 20.4 \\
\hline 2 & 41 & 16.21 & 44 & 17.6 & 2 & 48 & 18.97 & 43 & 17.2 \\
\hline 3 & 37 & 14.62 & 35 & 14 & 3 & 9 & 3.56 & 14 & 5.6 \\
\hline 4 & 6 & 2.37 & 8 & 3.2 & 4 & 1 & 0.4 & 8 & 3.2 \\
\hline Total & 252 & 99.6 & 250 & 100 & Total & 253 & 100 & 250 & 100 \\
\hline Neck & Freq & Percent & Freq & Percent & $\begin{array}{l}\text { Right hand/ } \\
\text { /arm/shoulder }\end{array}$ & Freq & Percent & Freq & Percent \\
\hline 0 & 109 & 43.08 & 100 & 40 & 0 & 90 & 35.57 & 54 & 21.6 \\
\hline 1 & 64 & 25.3 & 58 & 23.2 & 1 & 58 & 22.92 & 66 & 26.4 \\
\hline 2 & 62 & 24.51 & 65 & 26 & 2 & 67 & 26.48 & 79 & 31.6 \\
\hline 3 & 17 & 6.72 & 18 & 7.2 & 3 & 33 & 13.04 & 37 & 14.8 \\
\hline 4 & 1 & 0.4 & 9 & 3.6 & 4 & 5 & 1.98 & 14 & 5.6 \\
\hline Total & 253 & 100 & 250 & 100 & Total & 253 & 100 & 250 & 100 \\
\hline $\begin{array}{l}\text { Left hand/arm/ } \\
\text { /shoulder }\end{array}$ & Freq & Percent & Freq & Percent & Trunk & Freq & Percent & Freq & Percent \\
\hline 0 & 100 & 39.53 & 56 & 22.4 & 0 & 92 & 36.36 & 66 & 26.4 \\
\hline 1 & 59 & 23.32 & 61 & 24.4 & 1 & 55 & 21.74 & 79 & 31.6 \\
\hline 2 & 66 & 26.09 & 78 & 31.2 & 2 & 65 & 25.69 & 75 & 30 \\
\hline 3 & 25 & 9.88 & 46 & 18.4 & 3 & 40 & 15.81 & 25 & 10 \\
\hline 4 & 2 & 0.79 & 9 & 3.6 & 4 & 1 & 0.4 & 5 & 2 \\
\hline Total & 252 & 99.6 & 250 & 100 & Total & 253 & 100 & 250 & 100 \\
\hline $\begin{array}{l}\text { Right foot/leg/ } \\
\text { /hip }\end{array}$ & Freq & Percent & Freq & Percent & Left foot/leg/hip & Freq & Percent & Freq & Percent \\
\hline 0 & 88 & 34.78 & 76 & 30.4 & 0 & 109 & 43.08 & 68 & 27.2 \\
\hline 1 & 67 & 26.48 & 68 & 27.2 & 1 & 57 & 22.53 & 60 & 24 \\
\hline 2 & 69 & 27.27 & 65 & 26 & 2 & 61 & 24.11 & 83 & 33.2 \\
\hline 3 & 25 & 9.88 & 28 & 11.2 & 3 & 22 & 8.7 & 21 & 8.4 \\
\hline 4 & 4 & 1.58 & 13 & 5.2 & 4 & 4 & 1.58 & 18 & 7.2 \\
\hline Total & 253 & 100 & 250 & 100 & Total & 253 & 100 & 250 & 100 \\
\hline Communication & Freq & Percent & Freq & Percent & Drinking & Freq & Percent & Freq & Percent \\
\hline 0 & 72 & 28.46 & 114 & 45.6 & 0 & 81 & 32.02 & 70 & 28 \\
\hline 1 & 129 & 50.99 & 89 & 35.6 & 1 & 109 & 43.08 & 88 & 35.2 \\
\hline 2 & 44 & 17.39 & 38 & 15.2 & 2 & 50 & 19.76 & 56 & 22.4 \\
\hline 3 & 7 & 2.77 & 8 & 3.2 & 3 & 11 & 4.35 & 22 & 8.8 \\
\hline 4 & 1 & 0.4 & 1 & 0.4 & 4 & 2 & 0.79 & 14 & 5.6 \\
\hline Total & 253 & 100 & 250 & 100 & Total & 253 & 100 & 250 & 100 \\
\hline
\end{tabular}


Table 2 cont. Distribution of UDysRS responses

\begin{tabular}{lcccccccccc}
$\begin{array}{l}\text { Dressing } \\
\text { (objective) }\end{array}$ & \multicolumn{2}{c}{ Reference standard } & \multicolumn{2}{c}{ Polish } & \multicolumn{3}{c}{ Reference standard } & \multicolumn{2}{c}{ Polish } \\
\hline 0 & Freq & Percent & Freq & Percent & Ambulation & Freq & Percent & Freq & Percent \\
\hline 1 & 59 & 23.32 & 64 & 25.6 & 0 & 54 & 21.34 & 35 & 14 \\
2 & 76 & 30.04 & 71 & 28.4 & 1 & 99 & 39.13 & 86 & 34.4 \\
3 & 80 & 31.62 & 67 & 26.8 & 2 & 69 & 27.27 & 81 & 32.4 \\
4 & 28 & 11.07 & 30 & 12 & 3 & 29 & 11.46 & 36 & 14.4 \\
Total & 10 & 3.95 & 18 & 7.2 & 4 & 2 & 0.79 & 12 & 4.8 \\
& 253 & 100 & 250 & 100 & Total & 253 & 100 & 250 & 100
\end{tabular}

Table 3. Item-to-total correlations and Cronbach's alpha with deleted items

\begin{tabular}{|c|c|c|c|c|}
\hline \multirow[t]{3}{*}{ Deleted variables } & \multicolumn{4}{|c|}{ Cronbach Coefficient Alpha with Deleted Variable* } \\
\hline & \multicolumn{2}{|c|}{ Raw variables } & \multicolumn{2}{|c|}{ Standardised variables } \\
\hline & $\begin{array}{l}\text { Correlation** } \\
\text { with total }\end{array}$ & Alpha & $\begin{array}{l}\text { Correlation } \\
\text { with total }\end{array}$ & Alpha \\
\hline Q1 & 0.560 & 0.940 & 0.563 & 0.941 \\
\hline Q2 & 0.565 & 0.940 & 0.575 & 0.941 \\
\hline Q3 & 0.623 & 0.940 & 0.630 & 0.941 \\
\hline Q4 & 0.724 & 0.938 & 0.731 & 0.939 \\
\hline Q5 & 0.752 & 0.938 & 0.756 & 0.939 \\
\hline Q6 & 0.760 & 0.938 & 0.761 & 0.939 \\
\hline Q7 & 0.743 & 0.938 & 0.746 & 0.939 \\
\hline Q8 & 0.770 & 0.938 & 0.774 & 0.938 \\
\hline Q9 & 0.650 & 0.939 & 0.652 & 0.940 \\
\hline Q10 & 0.618 & 0.940 & 0.620 & 0.941 \\
\hline Q11 & 0.585 & 0.940 & 0.590 & 0.941 \\
\hline Q12 & 0.493 & 0.941 & 0.483 & 0.942 \\
\hline Q13 & 0.550 & 0.940 & 0.536 & 0.942 \\
\hline Q14 & 0.373 & 0.943 & 0.360 & 0.944 \\
\hline Q15 & 0.396 & 0.943 & 0.383 & 0.944 \\
\hline Q16 & 0.481 & 0.941 & 0.487 & 0.942 \\
\hline Q17 & 0.478 & 0.941 & 0.479 & 0.942 \\
\hline Q18 & 0.608 & 0.940 & 0.609 & 0.941 \\
\hline Q19 & 0.567 & 0.940 & 0.569 & 0.941 \\
\hline Q20 & 0.540 & 0.940 & 0.541 & 0.942 \\
\hline Q21 & 0.618 & 0.940 & 0.619 & 0.941 \\
\hline Q22 & 0.546 & 0.941 & 0.547 & 0.942 \\
\hline Q23 & 0.550 & 0.940 & 0.551 & 0.942 \\
\hline Q24 & 0.736 & 0.938 & 0.738 & 0.939 \\
\hline Q25 & 0.754 & 0.938 & 0.756 & 0.939 \\
\hline Q26 & 0.698 & 0.939 & 0.696 & 0.940 \\
\hline
\end{tabular}

Cronbach coefficient alpha is a measure of squared correlation between observed scores and true scores

*What Cronbach coefficient alpha would be if that variable were deleted

${ }^{*}$ Correlation between individual item and sum of remaining items 
Table 4. Exploratory Factor Analysis (EFA)

\begin{tabular}{|c|c|c|c|}
\hline \multirow[t]{2}{*}{ Factor } & \multirow[t]{2}{*}{ Item } & \multicolumn{2}{|c|}{ Item factor loading } \\
\hline & & $\begin{array}{l}\text { Reference } \\
\text { standard } \\
(n=246)\end{array}$ & $\begin{array}{c}\text { Polish } \\
(n=250)\end{array}$ \\
\hline \multirow[t]{14}{*}{ Factor 1} & Speech & 0.698 & 0.555 \\
\hline & Chewing/swallowing & 0.749 & 0.822 \\
\hline & Eating & 0.800 & 0.825 \\
\hline & Dressing & 0.861 & 0.875 \\
\hline & Hygiene & 0.825 & 0.773 \\
\hline & Handwriting & 0.780 & 0.862 \\
\hline & Doing hobbies/activities & 0.728 & 0.798 \\
\hline & Walking/balance & 0.731 & 0.827 \\
\hline & Public/social & 0.686 & 0.752 \\
\hline & Exciting situations & 0.718 & NA \\
\hline & Right hand/arm/shoulder & 0.412 & NA \\
\hline & Drinking & 0.441 & 0.479 \\
\hline & Dressing (objective) & 0.415 & 0.421 \\
\hline & Communication & NA & 0.473 \\
\hline \multirow[t]{15}{*}{ Factor 2} & Chewing/swallowing & 0.411 & NA \\
\hline & Walking/balance & 0.401 & NA \\
\hline & Public/social & 0.462 & NA \\
\hline & Face & 0.717 & 0.638 \\
\hline & Neck & 0.752 & 0.785 \\
\hline & Right hand/arm/shoulder & 0.701 & 0.739 \\
\hline & Left hand/arm/shoulder & 0.663 & 0.629 \\
\hline & Trunk & 0.769 & 0.703 \\
\hline & Right foot/leg/hip & 0.711 & 0.682 \\
\hline & Left foot/leg/hip & 0.741 & 0.728 \\
\hline & Communication & 0.775 & 0.704 \\
\hline & Drinking & 0.755 & 0.756 \\
\hline & Dressing (objective) & 0.739 & 0.662 \\
\hline & Ambulation & 0.729 & NA \\
\hline & Dystonia pain severity & NA & 0.591 \\
\hline \multirow[t]{4}{*}{ Factor 3} & Dystonia effects on activities & 0.883 & 0.978 \\
\hline & Effect of pain from dystonia & 0.971 & 0.966 \\
\hline & Dystonia pain severity & 0.945 & NA \\
\hline & Exciting situations & NA & 0.663 \\
\hline
\end{tabular}

$\mathrm{CFI}=0.942 ; \mathrm{RMSEA}=0.109 ; \mathrm{NA}-$ implies that listed item did not load on factor indicated loaded in factors 1 and 2 in the Polish version, but only in factor 2 in the Spanish version. Inversely, Right hand/arm/shoulder objective assessment of dyskinesia severity loaded on factors 1 and 2 in the Reference Standard, but only in factor 2 in the Polish version. Three items (Chewing/swallowing, Walking/balance, Public/social) loaded in factors 1 and 2 in the Reference Standard, but only in factor 1 in the Polish UDysRS version. Two items (Drinking and Dressing) loaded on more than one factor in both language versions.

\section{Discussion}

The Movement Disorders Society Rating Scales Programme leads the global translation effort of different assessment scales including the UDysRS. Currently, this programme includes 14 non-English validated editions of the UDysRS.

The original English version was clinimetrically evaluated to establish internal consistency and inter-rater reliability, but the small sample size of the English version precluded a comprehensive analysis of factor structure.

Because of this limitation, we could not compare the resultant structure from the present study with that of the original English version.

Therefore we instead compared our results to the Spanish version of the UDysRS. This was the first large-scale clinimetric analysis of this instrument, and is now recognised as the Reference Standard for UDysRS translations.

In agreement with the Reference Standard, the Polish version of the UDysRS demonstrated a clear factor structure, with three factors related to ON dyskinesia, OFF dystonia, and patient perceptions of the functional effects of dyskinesia. The overall factor structure of the Polish version was consistent with that of the Reference Standard. Exploratory factor analysis, where variability from sample to sample is expected, identified isolated, subtle item differences of factor structure between the Polish and the Reference Standard UDysRS.

We are aware that this study has some limitations related to potential sample selection bias.

The data comes from high reference neurology clinics specialising in movement disorders, and as such does not represent the general Polish PD patient population. However, this is a minor issue because neurologists at specialist centres are the most likely group to be using this scale for their research.

\section{Conclusions and clinical implications}

The Polish UDysRS was confirmed to share a common factor structure with the Reference Standard. Therefore, this version was designated to be the Official Polish version of the UDysRS, and will be available from the MDS website (https://www.movementdisorders.org/MDS-Files1/Education/Rating-Scales). 
In order to establish the UDysRS as the international 'gold standard' tool for the clinical assessment of dyskinesia in advanced Parkinson's Disease, fully tested non-English translations must be available for worldwide research programmes.

As such, having a validated Polish version of the scale will provide opportunities for Polish centres to contribute data from Polish-speaking individuals in large multicentre studies and clinical trials evaluating dyskinesia in PD patients.

Moreover, having the previously published validation of Polish translation of the MDS-UPDRS, plus now the validated Polish UDysRS version, allows movement disorders specialists in Poland to be fully equipped with two scales important in the objective assessment of moderate and severe Parkinson's Disease.

We believe that these tools will be useful in everyday clinical practice, especially in hospitals, where advanced treatment options (DBS surgery and infusion therapies) are available.

Worldwide use of the same rating measures (in appropriate translations) enhances international communication and offers a common ground on which to study similarities and differences in disease manifestations, progression, and disabilities across cultures.

Acknowledgements: We would like to thank our patients and their families for participating in this project. We are grateful to Shazia Ali for her assistance during the study preparation, in conducting and recapitulating the validation of the Polish translation of the MDS-UDysRS.

Funding: This publication was prepared without any external source of funding.

Conflicts of interest: The authors declare no conflict of interest.

\section{References}

1. Fabbrini G, Brotchie JM, Grandas F, et al. Levodopa-induced dyskinesias. Mov Disord. 2007; 22(10): 1379-89; quiz 1523, doi: 10.1002/ mds.21475, indexed in Pubmed: 17427940.

2. Espay AJ, Morgante F, Merola A, et al. Levodopa-induced dyskinesia in Parkinson disease: Current and evolving concepts. Ann Neurol. 2018;
84(6): 797-811, doi: 10.1002/ana.25364, indexed in Pubmed: 30357892.

3. Jost WH, Altmann C, Fiesel T, et al. Influence of levodopa on orthostatic hypotension in Parkinson's Disease. Neurol Neurochir Pol. 2020; 54(2): 200-203, doi: 10.5603/PJNNS.a2020.0019, indexed in Pubmed: 32219811.

4. Tipton PW, Uitti RJ, Cheshire WP. ,Falling off' the dopamine wagon. Neurol Neurochir Pol. 2019; 53(5): 390-391, doi: 10.5603/PJNNS. a2019.0048, indexed in Pubmed: 31657446.

5. Goetz CG, Stebbins GT, Chung KA, et al. Which dyskinesia scale best detects treatment response? Mov Disord. 2013; 28(3): 341-346, doi: 10.1002/mds.25321, indexed in Pubmed: 23390076.

6. Goetz CG, Nutt JG, Stebbins GT. The Unified Dyskinesia Rating Scale: presentation and clinimetric profile. Mov Disord. 2008; 23(16): 23982403, doi: 10.1002/mds.22341, indexed in Pubmed: 19025759.

7. Goetz CG, Stebbins GT, Wang Lu, et al. IPMDS-Sponsored Scale TransIation Program: Process, Format, and Clinimetric Testing Plan for the MDS-UPDRS and UDysRS. Mov Disord Clin Pract. 2014; 1(2): 97-101, doi: 10.1002/mdc3.12023, indexed in Pubmed: 27747259.

8. Cubo E, Goetz CG, Stebbins GT, et al. Independent Spanish Validation of the Unified Dyskinesia Rating Scale. Mov Disord Clin Pract. 2014; 1(3): 213-218, doi: 10.1002/mdc3.12065, indexed in Pubmed: 27891530.

9. Siuda J, Boczarska-Jedynak M, Budrewicz S, et al. Validation of the Polish version of the Movement Disorder Society-Unified Parkinson's Disease Rating Scale (MDS-UPDRS). Neurol Neurochir Pol. 2020; 54(5): 416-425, doi: 10.5603/PJNNS.a2020.0049, indexed in Pubmed: 32639019.

10. Fowler FJ. Improving survey questions: design and evaluation. Applied social research methods series. 38. Thousand Oaks, CA: Sage Publications. ; 1995.

11. Muthen LK. and Muthen BO. M plus user's guide. Sixth edition. Los Angeles, CA: Muthen. : Muthen.

12. Hatcher L. Step-by-step approach to using the SAS system for factor analysis and structural equation modeling. 1994. SAS Inst. , Cary, NC.; 73.

13. Brown TA. Confirmatory Factor Analysis for Applied Research. New York, NY: The Guilford Press. SAGE Publications. : Inc.

14. Browne M. An Overview of Analytic Rotation in Exploratory Factor Analysis. Multivariate Behavioral Research. 2010; 36(1): 111-150, doi: 10.1207/s15327906mbr3601_05.

15. Gorsuch RL. Factor analysis. Second edition. Hillsdale, NJ: 1983. Lawrence Erlbaum Associations. : Inc. 